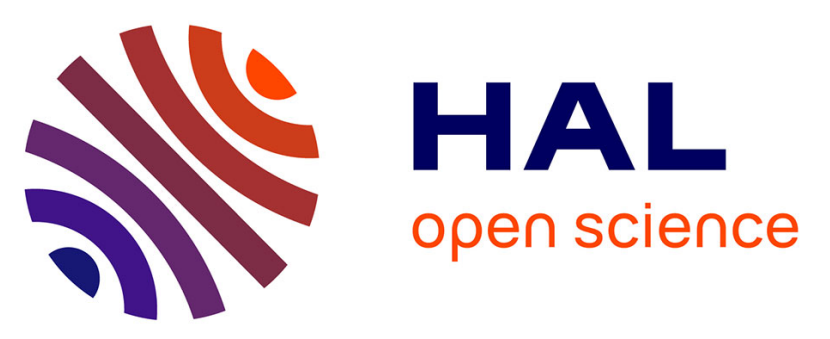

\title{
Influence of high numerical aperture on depth-of-field enhancing phase mask optimization in localization microscopy
}

Olivier Lévêque, Romain Duverger, Hervé Sauer, Caroline Kulcsár, François Goudail

\section{To cite this version:}

Olivier Lévêque, Romain Duverger, Hervé Sauer, Caroline Kulcsár, François Goudail. Influence of high numerical aperture on depth-of-field enhancing phase mask optimization in localization microscopy. Journal of the Optical Society of America. A Optics, Image Science, and Vision, 2021, 38 (9), pp.13801390. 10.1364/JOSAA.432696 . hal-03331525

\section{HAL Id: hal-03331525 \\ https://hal.science/hal-03331525}

Submitted on 1 Sep 2021

HAL is a multi-disciplinary open access archive for the deposit and dissemination of scientific research documents, whether they are published or not. The documents may come from teaching and research institutions in France or abroad, or from public or private research centers.
L'archive ouverte pluridisciplinaire HAL, est destinée au dépôt et à la diffusion de documents scientifiques de niveau recherche, publiés ou non, émanant des établissements d'enseignement et de recherche français ou étrangers, des laboratoires publics ou privés. 


\title{
Influence of high numerical aperture on depth-of-field enhancing phase mask optimization in localization microscopy
}

\author{
Olivier LÉvêque ${ }^{1,}$, Romain Duverger ${ }^{1}$, Hervé Sauer ${ }^{1}$, Caroline Kulcsár ${ }^{1}$, and François \\ GOUDAIL $^{1}$ \\ ${ }^{1}$ Université Paris-Saclay, Institut d'Optique Graduate School, CNRS, Laboratoire Charles Fabry, 91127, Palaiseau, France. \\ *Corresponding author: olivier.leveque@institutoptique.fr \\ Compiled September 1, 2021
}

\begin{abstract}
The depth-of-field (DoF) of localization microscopes can be extended by placing a phase mask in the aperture stop of the objective. To optimize these masks and characterize their performance, defocus is in general modeled by a simple quadratic pupil phase term. However, this model does not take into account two essential characteristics of localization microscopy setups: extremely high numerical aperture (NA) and mismatch between the refractive indices of immersion liquid and sample. Using the more realistic high NA image formation model of Gibson \& Lanni (GL), we show that DoF extension is simply reduced by a NA-dependent scaling factor. We also show that, provided this scaled DoF extension factor is taken into account, masks optimized with the approximate quadratic model are still nearly optimal in the framework of the GL model. This result is important since it establishes that generic optimized masks can be used in setups with different NA and immersion indices. (๑) 2021 Optical Society of America. One print or electronic copy may be made for personal use only. Systematic reproduction and distribution, duplication of any material in this paper for a fee or for commercial purposes, or modifications of the content of this paper are prohibited.
\end{abstract}

\section{INTRODUCTION}

The depth-of-field (DoF) of localization microscopes is limited by the natural DoF of the objective, which is very short since the numerical aperture (NA) must be large to increase lateral resolution. Several approaches have been investigated in order to improve the DoF, involving multi-focusing techniques [1] or phase masks $[2,3]$. Among them, we have proposed a method based on annular binary phase masks, which are easy to manufacture. After proper optimization and appropriate signal processing, they have been shown to be able to improve the DoF by a factor $3[4,5]$.

To optimize these masks and characterize their performance, the defocus wavefront aberration is in general modeled as a quadratic function of the pupil coordinates [6]. However, this model, which we will call "quadratic approximation" (QA), does not include two essential features of microscope objectives used for localization microscopy: extremely high NA and possible mismatch between nominal immersion liquid and true sample refractive indices. There exists more accurate scalar or vectorial models that better account for these imaging characteristics. Among them, the "Gibson \& Lanni" (GL) scalar model [7] is significantly more accurate than the QA model for computing the actual point spread function (PSF) variation with defocus, while being much more easy to tackle than more complex vectorialbased models $[8,9]$.

An important question in practice is to determine if masks optimized with the approximate QA model are also efficient in the more realistic world described by the GL model. The purpose of the present article is to investigate this issue. A major difficulty is that in the QA model, the effect of defocus can be entirely described with a single reduced parameter $\psi$ (the peak-to-valley quadratic defocus wavefront error), whereas in the GL model, it depends on several parameters (NA, mismatches between real and nominal focusing depths and refractive indices). To address this difficulty, we first show that although the DoF predicted by the QA and the GL models are significantly different, they can be connected by a simple scaling factor that depends on the NA and on the sample refractive index. Second, we compare the optimization of DoF enhancing annular binary phase masks for these equivalent configurations in the QA and GL models. We show that the resulting optimal masks are similar and yield similar localization performance. This result is important since it makes it possible to use generic phase masks that are nearly optimal for many different configurations of NA, focusing depth and index mismatch. 


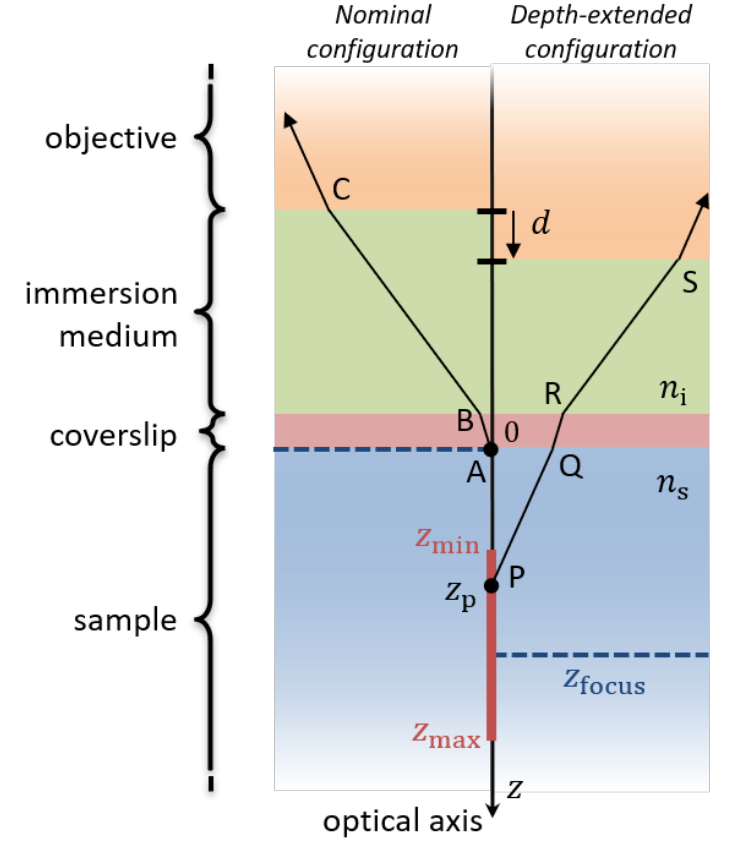

Fig. 1. Schematic drawing of the nominal and depth-extended object-space configurations of the immersion microscope objective. $A B C$ is a ray originating from a point source located just below the coverslip when $d$ has its nominal 0 value. PQRS is a ray from a point source located within the targeted DoF range $\left[z_{\min }, z_{\max }\right]$ when $d \neq 0$.

The article is organized as follows. In Section 2, we define the defocused microscope setup investigated in this paper and its parameters. In Section 3, we introduce DoF enhancing with annular binary phase masks and their optimization in the framework of the QA model. In Section 4, we determine the equivalent expressions of the DoF for the QA and GL models as a function of imaging parameters. In Section 5, we show that for these equivalent DoF settings, the optimal masks in the QA and GL models are similar and yield similar localization performance. Section 6 is devoted to conclusions and perspectives to this work.

\section{DESCRIPTION OF MICROSCOPE CONFIGURATION IN THE PRESENCE OF DEFOCUS}

We consider in this paper an immersion microscope objective (associated with the microscope tube lens if it is infinity corrected) that gives an image with lateral magnification $M$ and which is assumed to be, as usual for such lens, object-space telecentric, aplanatic (i.e., it fulfills the Abbe sine condition), and with negligible on-axis and field aberrations when used in its nominal configuration. As shown on the left side of Fig. 1, the objective is nominally designed to work immersed in a liquid of refractive index $n_{\mathrm{i}}$ with a given coverslip and a sample observed just below the coverslip. DoF extension will be used to image objects that stand below the coverslip in a medium of refractive index $n_{\mathrm{s}}$ (see the right side of Fig. 1). In this section and the next one, we will assume that $n_{\mathrm{i}}=n_{\mathrm{s}}$; possible index mismatch between the two mediums will be considered in subsequent sections.

Let us denote the 3D position of a fluorescent emitter in the observed sample by the coordinates $\boldsymbol{\theta}=\left(x_{\mathrm{p}}, y_{\mathrm{p}}, z_{\mathrm{p}}\right)$, and the $2 \mathrm{D}$ position of a point of observation in the image plane (i.e., on the image sensor) by the coordinates $(x, y)$. Due to object-space telecentricity, the magnification $M$ does not depend on the $z_{\mathrm{p}}$ value. Assuming the field aberrations are negligible, this leads to a space-invariant $(x, y)$ response where the irradiance in the image plane of a point like emitter can be written:

$$
f(x, y, \boldsymbol{\theta})=h_{z_{\mathrm{p}}}\left(x-M x_{\mathrm{p}}, y-M y_{\mathrm{p}}\right)
$$

where $h_{z_{\mathrm{p}}}(x, y)$ is the point spread function (PSF) of the microscope objective, i.e., the image-plane irradiance of an emitter centered at lateral position $\left(x_{\mathrm{p}}, y_{\mathrm{p}}\right)=(0,0)$ and located at a depth $z_{\mathrm{p}}$. Due to the large value of the magnification $|M|$, the image-space numerical aperture, denoted by $\mathrm{NA}_{i}$, is linked to the object-space numerical aperture, denoted by NA, by the Abbe sine condition: $\mathrm{NA}_{\mathrm{i}}=\mathrm{NA} /|M|$. Since $\mathrm{NA}_{\mathrm{i}}$ is small, classical paraxial Fourier Optics can be applied to compute the PSF in the image plane [6]:

$$
h_{z_{\mathrm{p}}}(x, y) \propto\left|\mathrm{FT}_{2 \mathrm{D}}\left[\Pi_{\text {Disk }} \exp \left(i \Phi_{z_{\mathrm{p}}}\right)\right]\left(x \frac{\mathrm{NA}_{\mathrm{i}}}{\lambda}, y \frac{\mathrm{NA}_{\mathrm{i}}}{\lambda}\right)\right|^{2}
$$

where the operator $\mathrm{FT}_{2 \mathrm{D}}$ denotes the $2 \mathrm{D}$ Fourier transform, $\lambda$ is the wavelength of the collected light, $\Pi_{\text {Disk }}$ is equal to 1 inside the exit pupil disk (of normalized radius 1 ) and 0 elsewhere, and $\Phi_{z_{\mathrm{p}}}$ is the exit pupil phase function. $\Pi_{\text {Disk }}$ and $\Phi_{z_{\mathrm{p}}}$ are assumed to be $2 \mathrm{D}$ circular symmetric functions of the normalized exit pupil coordinates. Note that $h_{z_{\mathrm{p}}}(x, y)$ can be expressed using the object-space NA by replacing $\mathrm{NA}_{i} / \lambda$ by NA $/(|M| \lambda)$. We assume that the function $f(x, y, \theta)$ is normalized so that $\iint f(x, y, \boldsymbol{\theta}) \mathrm{d} x \mathrm{~d} y=1$. The pupil phase function is given by

$$
\Phi_{z_{\mathrm{p}}}(r)=\frac{2 \pi}{\lambda} W_{z_{\mathrm{p}}}(r)
$$

where $W_{z_{\mathrm{p}}}(r)$ denotes the optical path difference (OPD) between the focused and defocused spherical wavefronts at a normalized radius $r$ in the exit pupil. The above mentioned QA and GL models correspond to different expressions of $W_{z_{p}}(r)$.

\section{DOF ENHANCING PHASE MASK OPTIMIZATION}

In this section, we describe the optimization of DoF enhancing phase masks when the usual QA model is used for representing the PSF variation with defocus.

\section{A. Defocus within quadratic approximation model}

For an instrument limited by diffraction in the presence of pure defocus, one often uses the following quadratic approximation of the OPD [6]:

$$
W_{\mathrm{QA}}(r)=\psi r^{2} \text {. }
$$

Note that to simplify notation, we have dropped the subscript $z_{\mathrm{p}}$ in the expression of the OPD. In Eq. (4), the parameter $\psi$ represents the value of the first non-zero expansion coefficient of the OPD between the focused and defocused spherical wavefronts at the edge of the pupil and is suitable for low or moderate NA. We assume in this section no mismatch between sample and immersion medium indices. The parameter $\psi$ has the following expression $[6,10]$ :

$$
\psi=\frac{\mathrm{NA}^{2}}{2 n_{\mathrm{s}}}\left(z_{\mathrm{p}}-z_{\text {focus }}\right)
$$

where $n_{\mathrm{s}}=n_{\mathrm{i}}$ is the matched refractive index of the sample and immersion media, $z_{p}$ is the actual position of the fluorescent emitter along the optical axis and $z_{\text {focus }}$ the position at which 
the instrument is focused. If $z_{\mathrm{p}}=z_{\text {focus, }}$, then $W_{\mathrm{QA}}(r)=0$, the wavefront error vanishes, and the PSF is described by the well known Airy pattern centered at the ideal image position:

$$
f_{\text {Airy }}(x, y, \boldsymbol{\theta}) \propto\left|\frac{J_{1}\left(\frac{2 \pi \mathrm{NA}}{|M| \lambda} \sqrt{\left(x-|M| x_{\mathrm{p}}\right)^{2}+\left(y-|M| y_{\mathrm{p}}\right)^{2}}\right)}{\frac{2 \pi \mathrm{NA}}{|M| \lambda} \sqrt{\left(x-|M| x_{\mathrm{p}}\right)^{2}+\left(y-|M| y_{\mathrm{p}}\right)^{2}}}\right|^{2}
$$

where $J_{1}$ denotes the Bessel function of the first kind of order 1 .

Let us denote by $\left[z_{\min }, z_{\max }\right]$ the targeted DoF range, that is, the range of values of $z_{\mathrm{p}}$ within which we want the localization to be accurate. In the QA model, since the deviation of the defocused PSF from the Airy pattern depends only on the defocus parameter $\psi$, the targeted DoF range can be entirely specified with this reduced parameter. Indeed, in order to have the sharpest PSF, we have to minimize the maximum value of $|\psi|$, i.e., of the phase aberration function $\Phi_{\mathrm{QA}}(r)$, reached when $z_{\mathrm{p}}$ varies over the targeted DoF range. Obviously, this is obtained by setting the focus point at the middle distance:

$$
z_{\text {focus }}^{\text {opt }}=\frac{z_{\max }+z_{\min }}{2}
$$

With this setting, the maximum absolute value of $\psi$ is reached at the ends of the DoF range. Indeed, from Eq. (5), the defocus parameter is equal to $-\psi_{\max }$ at $z_{\mathrm{p}}=z_{\min }$ and to $\psi_{\max }$ at $z_{\mathrm{p}}=$ $z_{\text {max }}$ with

$$
\psi_{\max }=\frac{\mathrm{NA}^{2}}{4 n_{\mathrm{s}}}\left(z_{\max }-z_{\min }\right) .
$$

Within the targeted DoF range, the defocus parameter $\psi$ thus varies in the interval $\left[-\psi_{\max }, \psi_{\max }\right]$.

As an illustration, we have represented in Fig. 2.a the variation of the PSF profile as a function of $\psi$, for the microscope configuration described in Table 1. Note that since the PSF has circular symmetry, a cross-section along any line passing through its center is sufficient to represent its properties. The left-side vertical axis in Fig. 2.a represents the defocus parameter $\psi$ expressed in units of the wavelength $\lambda$ of the collected light, the graph being drawn for $\psi \in[-1.5 \lambda, 1.5 \lambda]$. The right-side vertical axis represents the corresponding axial emitter position $z_{\mathrm{p}}$. The relationship between these two quantities is given by Eq. (5) with $z_{\text {focus }}=z_{\text {focus }}^{\text {opt }}$. We observe on the graph that the farther the emitter is from the in-focus plane (i.e., the larger $|\psi|$ is), the more the PSF spreads out with its central lobe getting fainter. This greatly limits the localization accuracy of out-offocus fluorescent emitters.

\section{B. Localization accuracy evaluation using the Cramér-Rao bound}

The primary interest in localization microscopy is the localization accuracy of fluorescent emitters over a given range of defocus values. This accuracy can be expressed by the Cramér-Rao bound (CRB), which is a lower bound on the estimation variance that can be reached with an unbiased localization algorithm [11]. In the following, we will rather consider its square root, denoted by RCRB, since this value has the dimension of a standard deviation and is a length that will be expressed in nanometers in this article.

The value of the RCRB depends on the nature and the level of the noise sources that corrupt the observed images [12]. The three main sources of noise occurring in modern sensors are photon noise (i.e., shot noise), dark noise, and read-out noise. With high-performance cameras, the latter two noise sources

\begin{tabular}{lcc}
\hline Simulation parameters & Symbols & Values \\
\hline Fluorophore position in the plane & $\left(x_{\mathrm{p}}, y_{\mathrm{p}}\right)$ & $(0,0) \mu \mathrm{m}$ \\
Fluorophore wavelength emission & $\lambda$ & $700 \mathrm{~nm}$ \\
Total number of photo-electrons & $N_{0}$ & $500{\mathrm{ph} . \mathrm{e}^{-}}$ \\
PSF image length & $2 P+1$ & 21 pixels \\
Pixel length & $\Delta_{x y}$ & $10 \mu \mathrm{m}$ \\
Object numerical aperture & $\mathrm{NA}$ & 1.3 \\
Lateral magnification & $M$ & 60 \\
Biological sample refractive index & $n_{\mathrm{s}}$ & 1.33 \\
Immersion refractive index & $n_{\mathrm{i}}$ & 1.33 or 1.52 \\
\hline${ }^{*}$ We consider two types of nominal immersion medium: water or oil.
\end{tabular}

Table 1. Microscope configuration and simulation parameters used in the present paper.

can often be considered negligible [8]. Photon noise can arise from the useful fluorescence signal (i.e., the light emitted by the excited molecule) or from the scene background. The level of background noise depends on the application, and in the present paper, for the sake of simplicity, we will neglect it, considering only the shot noise due to the light emitted by the excited molecule. Note, however, that our methodology and our main conclusions can be easily generalized to the presence of background shot noise.

As a reminder, $\boldsymbol{\theta}=\left(x_{\mathrm{p}}, y_{\mathrm{p}}, z_{\mathrm{p}}\right)$ is the 3D position of a fluorescent emitter and $(x, y)$ is the $2 \mathrm{D}$ position of its image on the sensor. Due to the quasi-circular symmetry of the 2D pixelated spatial distribution of irradiance (around its centroid) on the sensor for any value of $z_{\mathrm{p}}$, the non-diagonal terms of the Fisher information matrix [11] are negligible. The RCRB values along the $x$ and $y$-axis directions are therefore simply equal to the inverse square root of its diagonal terms. According to Ref. [4], the RCRB also depends on the sub-pixel position of the PSF relatively to the pixel grid (i.e., the "phasing"). The largest value of the RCRB (which corresponds to the worst localization precision) is obtained when the PSF is centered on a pixel of the sensor. In this case, and in the presence of emitter shot noise only, the RCRB along the $x$ and $y$-axis directions has the same value and can be calculated as follows [4]:

$$
\mathrm{RCRB}=\left[N_{0} \sum_{i=-P}^{P} \sum_{j=-P}^{P} \frac{\left(\int_{\left(j-\frac{1}{2}\right) \Delta_{x y}}^{\left(j+\frac{1}{2}\right) \Delta_{x y}} \Delta f_{i}\left(y, \boldsymbol{\theta}_{0}\right) \mathrm{d} y\right)^{2}}{\int_{\left(i-\frac{1}{2}\right) \Delta_{x y}}^{\left(i+\frac{1}{2}\right) \Delta_{x y}} \int_{\left(j-\frac{1}{2}\right) \Delta_{x y}}^{\left(j+\frac{1}{2}\right) \Delta_{x y}} f\left(x, y, \boldsymbol{\theta}_{0}\right) \mathrm{d} x \mathrm{~d} y}\right]^{-\frac{1}{2}}
$$

where $N_{0}$ denotes the total number of photo-electrons expected in the squared observed image of $(2 P+1)^{2}$ pixels, $\Delta_{x y}$ the pitch of the pixel grid (assuming a fill factor of $100 \%)$ and $\Delta f_{i}\left(y, \theta_{0}\right)$ the difference between the PSF values at the opposite edges of the pixels in row $i$ :

$$
\Delta f_{i}\left(y, \boldsymbol{\theta}_{0}\right)=f\left(\left[i-\frac{1}{2}\right] \Delta_{x y}, y, \boldsymbol{\theta}_{0}\right)-f\left(\left[i+\frac{1}{2}\right] \Delta_{x y}, y, \boldsymbol{\theta}_{0}\right)
$$

with $\theta_{0}=\left(0,0, z_{p}\right)$. It is clear from Eq. (9) that the accuracy with which an emitter can be located depends on the shape of the PSF, and thus on the value of the defocus parameter $\psi$. Indeed, we have represented in Fig. 2.c (blue solid line) the value of the RCRB as a function of $\psi$. We observe that the RCRB 


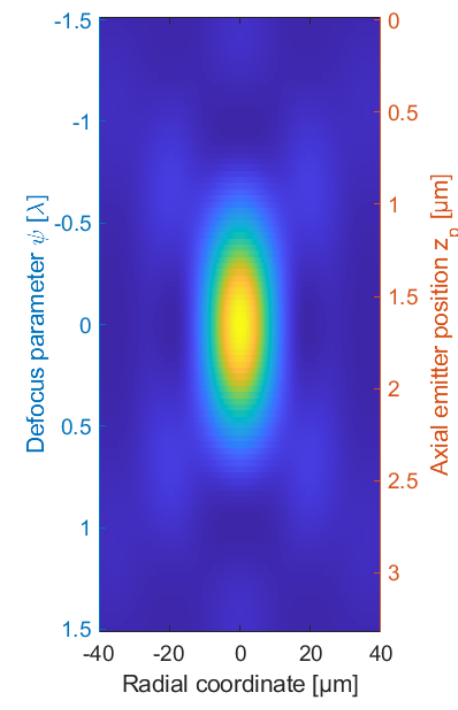

(a)

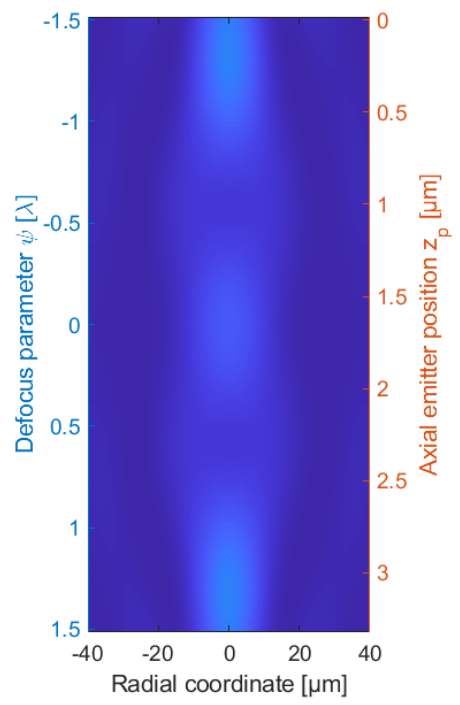

(b)

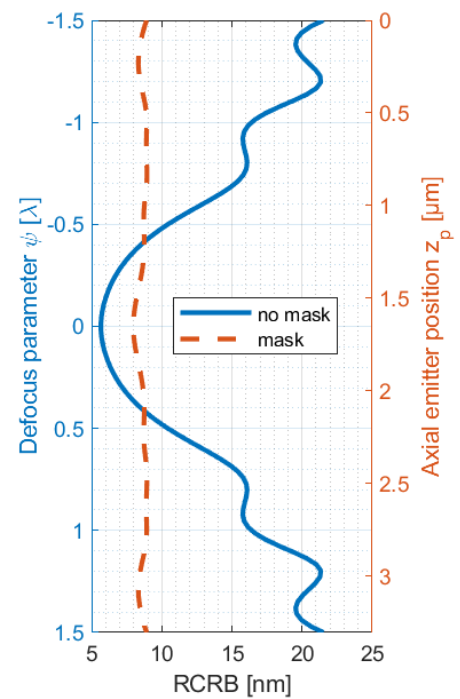

(c)

Fig. 2. Variation of the PSF profile, in the QA framework, as a function of the defocus parameter $\psi \in[-1.5 \lambda, 1.5 \lambda]$ or the axial emitter coordinates $z_{\mathrm{p}}$ (a) for an aberration-free diffraction-limited system with a circular aperture, (b) for the same optical system using an optimal binary phase mask. (c) Curves of the localization accuracy (i.e., the RCRB value) without (blue solid line) or with (red dashed line) phase mask. The microscope configuration and the simulation parameters are given in Table 1 (with here $n_{\mathrm{i}}=$ $\left.n_{\mathrm{s}}=1.33\right)$.

increases very quickly as the PSF degrades because the DoF of the system is small. We will quantify this degradation precisely in Section 4.B.

\section{Enhancing DoF with phase masks}

As proposed in Ref. [5], a solution to improve the RCRB over the targeted DoF range is to introduce in its aperture stop a phase function $\Phi_{\text {mask }}$ to modify the PSF of the microscope objective, such that Eq. (3) becomes:

$$
\Phi_{z_{\mathrm{p}}}(r, \rho)=\frac{2 \pi}{\lambda} W_{z_{\mathrm{p}}}(r)+\Phi_{\text {mask }}(r, \rho)
$$

where the vector $\rho$ denotes the parameters that define the mask. Note that although the results presented in this article are limited to phase masks with circular symmetry, they can easily be extended to the many types of phase masks proposed in the literature to improve the DoF [13-18]. As already mentioned, annular binary phase masks are easy to manufacture and they have been shown to extend DoF in localization microscopy [4,5]. This type of mask is based on concentric rings implementing static spatial phase modulations of alternatively 0 and $\pi$ radians at a nominal wavelength $\lambda$ as illustrated in Fig. 3. An $N$-ring phase mask is entirely characterized by the vector $\rho=\left(\rho_{1}, \rho_{2}, \cdots, \rho_{N-1}\right)$ of the normalized radii of each ring. The last radius is at the edge of the aperture, i.e., $\rho_{N}=1$. Our goal is to find the optimal mask, that is, the optimal parameter vector $\rho_{\text {opt }}$, that minimizes the RCRB over the defocus range. In other words,

$$
\rho_{\mathrm{opt}}=\arg \min _{\rho} J(\rho)
$$

with

$$
J(\boldsymbol{\rho})=\max _{\psi \in\left[-\psi_{\max }, \psi_{\max }\right]} \operatorname{RCRB}(\rho, \psi)
$$

where we have explicitly indicated the dependence of the RCRB in $\rho$ and $\psi$. For illustration, let us consider the optimal annular binary phase mask for a targeted DoF range of $\psi_{\max }=1.5 \lambda$

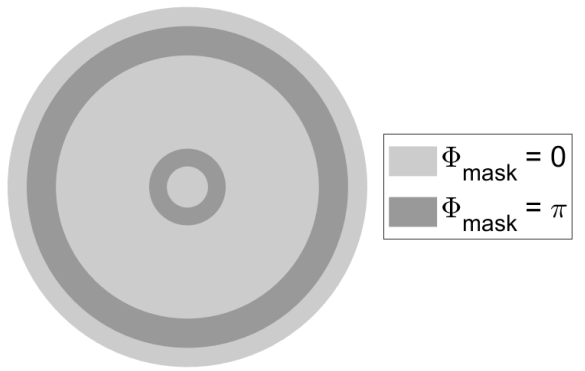

Fig. 3. Example of a 5-ring annular binary phase mask. This mask is optimal for a targeted DoF range of $\psi_{\max }=1.5 \lambda$ in the QA framework.

obtained by solving Eq. (12) as explained in Ref. [4]. The phase modulation of this mask is illustrated in Fig. 3. We have represented the variation of the PSF profile using this optimal mask in Fig. 2.b and the corresponding RCRB as a red dashed line in Fig. 2.c. We note that when a mask is used, the RCRB is much more invariant to defocus and significantly smaller for large defocus values.

This mask has been optimized under the assumption that the defocus-dependent OPD, and thus the PSF, is given by the QA model. As mentioned previously, this model is physically suitable for diffraction-limited microscope objectives with moderate values of NA and a perfect match between refractive indices. For higher NA values and a possible mismatch between the refractive indices, this model therefore becomes inadequate. There exists more accurate physical imaging models for representing outof-focus emitters in real microscopy applications $[9,10,19,20]$. We will focus on the Gibson \& Lanni model, a simple and efficient scalar model to describe the PSF of high NA immersion microscope objectives [7]. The question we will investigate is whether this more realistic model leads to different optimal masks and different DoF enhancing performance. 


\section{THE GIBSON \& LANNI MODEL FOR OUT-OF-FOCUS EMITTERS}

Modern microscope objectives are designed with high NA and provide optimal imaging conditions for a point-source located immediately below the coverslip (i.e., $z_{\mathrm{p}}=0$ ). However, these conditions are no longer met as soon as the emitters are located a few micrometers below the coverslip since extremely high NA and refractive index mismatch can give rise to non-negligible spherical aberrations of third or higher orders.

\section{A. The Gibson \& Lanni model}

We use the GL model to better account for the imaging characteristics described above [7]. This model considers that the imaging conditions are ideal if the emitter is located just below the coverslip and the optical parameters (including the coverslip thickness and index) have their nominal values. All the observed aberrations thus arise from factors external to the microscope objective, such as displacement of the emitter or variation of the optical parameters. In the present article, we assume that the values of the refractive indices and the microscope settings are perfectly known, and that the coverslip has its nominal properties. Under these assumptions, the OPD or wavefront error has, according to Ref. [7], the following expression:

$$
\begin{aligned}
W_{\mathrm{GL}}(r)=d n_{\mathrm{i}} & {\left[\sqrt{1-\left(\frac{\mathrm{NA} r}{n_{\mathrm{i}}}\right)^{2}}-1\right] } \\
& -z_{\mathrm{p}} n_{\mathrm{s}}\left[\sqrt{1-\left(\frac{\mathrm{NA} r}{n_{\mathrm{s}}}\right)^{2}}-1\right],
\end{aligned}
$$

where $n_{\mathrm{s}}, n_{\mathrm{i}}, z_{\mathrm{p}}$ and $d$ have been defined in Section 2 and in Fig. 1, and $r$ is the normalized radial coordinate on the entrance or exit pupil that ranges between 0 and 1 . Note that if $z_{p}=0$ and $d=0$ (i.e., the emitter is located immediately below the coverslip and in-focus), then $W_{\mathrm{GL}}(r)=0$ and the PSF is described by the Airy pattern, see Eq. (6). We can also note that the QA model in Eq. (4) corresponds to an approximation of the GL model defined in Eq. (14) when $n_{\mathrm{i}}=n_{\mathrm{s}}$ and $\mathrm{NA} \ll 1$.

Let us assume that the targeted DoF is $\left[z_{\min }, z_{\max }\right]$ as illustrated in Fig. 1. The parameter $d$ can be adjusted to focus the microscope at any point within the sample, and the question is how to set this focus in order to have the sharpest PSF over the whole DoF range. This question is more difficult to answer than in the QA case since there is no reduced parameter which entirely characterizes the OPD expression of Eq. (14). To solve this problem, we choose to represent the global amplitude of the OPD by its quadratic deviation $\sigma_{\mathrm{GL}}$ over the whole pupil. We call it the root mean square (RMS) wavefront error, and it is precisely defined in Appendix A. To have the sharpest PSF, the parameter $d$ must be chosen so as to minimize the maximum value of $\sigma_{\mathrm{GL}}$ over the targeted DoF range. In Appendix B, we determine this optimal value $d_{\mathrm{opt}}$, and show that it leads to focus the microscope at the distance $z_{\mathrm{p}}=z_{\text {focus }}^{\text {opt }}$, defined in Eq. (7). Interestingly, the optimal focusing distance is thus the same for the QA and GL models, i.e., halfway between $z_{\min }$ and $z_{\max }$.

\section{B. The conditions of approximate equivalence between the GL and QA models}

In this section, we compare the imaging properties predicted by the QA and the GL models. We have represented in Fig. 4.b the variation of the PSF profile as a function of the axial emitter position $z_{\mathrm{p}}$ when $d=d_{\text {opt }}$. The microscope configuration and the simulation parameters are given in Table 1 , and $n_{\mathrm{i}}=n_{\mathrm{s}}=$ 1.33. It is observed that this graph is quite similar to the graph obtained with the QA model represented in Fig. 4.a (this is the same figure as Fig. 2.a, it has been reproduced here to facilitate comparison). The main difference between Fig. 4.b and Fig. 4.a is a global "shrinking" of the PSF variation with the GL model. This means that the natural DoF predicted by the GL model is smaller than the one predicted by the QA model.

To confirm this interpretation, we have represented in Fig. 4.d, the Strehl ratio for the QA model (red solid line) and the GL model (blue solid line). The Strehl ratio is defined as the ratio between the maximal value of the PSF at depth $z_{p}$ and the maximal value of the Airy pattern. We notice in Fig. 4.d that the curves corresponding to the QA and GL models have similar shapes and can be related by a simple scaling factor. In other words, it appears that the PSF provided by the GL model for a given value of $z_{\mathrm{p}}$ is quasi-equal to the PSF given by the QA model, but for a value of $\psi$ different from that defined in Eq. (5). We show in Appendices C and D that this "equivalent" value of $\psi$ is equal to:

$$
\psi^{*}=\kappa_{\psi}\left(z_{\mathrm{p}}-z_{\text {focus }}^{\mathrm{opt}}\right)
$$

where

$$
\kappa_{\psi} \simeq \frac{\mathrm{NA}^{2}}{2 n_{\mathrm{s}}}+\frac{\mathrm{NA}^{4}}{8 n_{\mathrm{s}}^{3}}+\frac{11 \mathrm{NA}^{6}}{192 n_{\mathrm{s}}^{5}}+\frac{25 \mathrm{NA}^{8}}{768 n_{\mathrm{s}}^{7}} .
$$

It is easily seen that $\psi^{*}$ is always larger than that the value of $\psi$ given by the QA model in Eq. (5), which means that the actual defocus in the GL model is larger than the one predicted by the QA model. The difference between these two values increases with NA.

To illustrate this result, we have represented in Fig. 4.c the variation of the PSF profile computed with the QA model as a function of $\psi^{*}$ (this is a scaled version of Fig. 4.a). It is clear that Fig. 4.c is now very similar to Fig. 4.b: with this definition of the equivalent defocus parameter $\psi^{*}$, the PSF profiles given by the QA and the GL models are quasi-equal. This result is confirmed by plotting the corresponding values of the Strehl ratio in Fig. 4.d (red dashed curve): it is nearly superimposed on the GL model (blue solid curve).

Let us now consider the case where $n_{\mathrm{i}} \neq n_{\mathrm{s}}$. We have plotted in Fig. 5 the same graphs as in Fig. 4.b, 4.c and 4.d, but for $n_{\mathrm{i}}=1.52$ and $n_{\mathrm{s}}=1.33$. It is seen by comparing the blue and red solid curves in Fig. 5.c that most of the deviation between the Strehl ratios of the QA and GL models still amounts to a scaling. However, the fact that $n_{\mathrm{i}} \neq n_{\mathrm{S}}$ introduces two differences compared with the previous case. The first one is that the Strehl ratio of the GL model at the best focus point is no longer equal to one (see the blue solid curve in Fig. 5.c). This is due to the fact that when $n_{\mathrm{i}} \neq n_{\mathrm{s}}$, the RMS wavefront error is non zero even at $z_{\mathrm{p}}=z_{\text {focus }}$ (see Eq. (27)). The expression of the correcting factor $\beta$ on the Strehl ratio is given in Eq. (34). The second difference is that the PSF variation is now slightly asymmetric with respect to the focus point. This is due to the fact that in case of index mismatch, the wavefront error $W_{\mathrm{GL}}(r)$ over the pupil, defined in Eq. (14), has an asymmetrical behavior around $z_{\text {focus }}$ even if the RMS wavefront error is perfectly symmetric, as shown by Eq. (27).

We have represented in Fig. 5.b the variation of the PSF of the QA model as a function of the equivalent parameter $\psi^{*}$ and multiplied by the correcting factor $\beta$, which is equal to $\beta=0.89$ in our case. We have also plotted in Fig. 5.c the corresponding value of the Strehl ratio multiplied by $\beta$ (red dashed curve). It is 


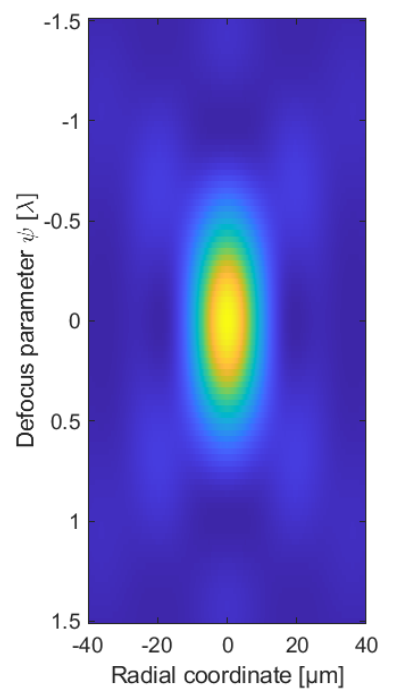

(a)

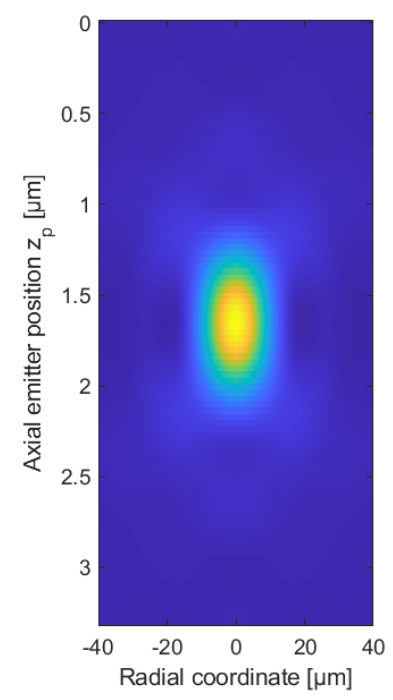

(b)

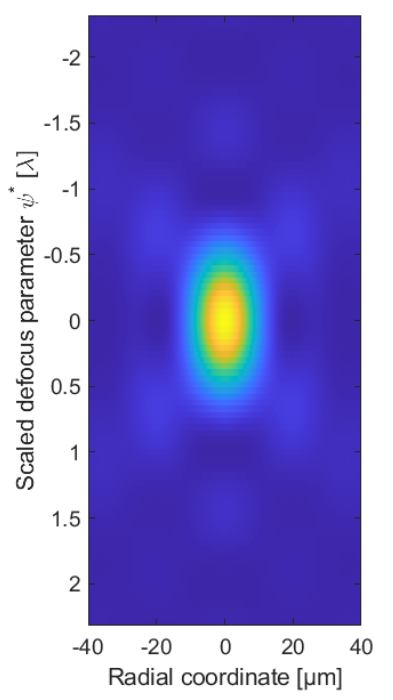

(c)

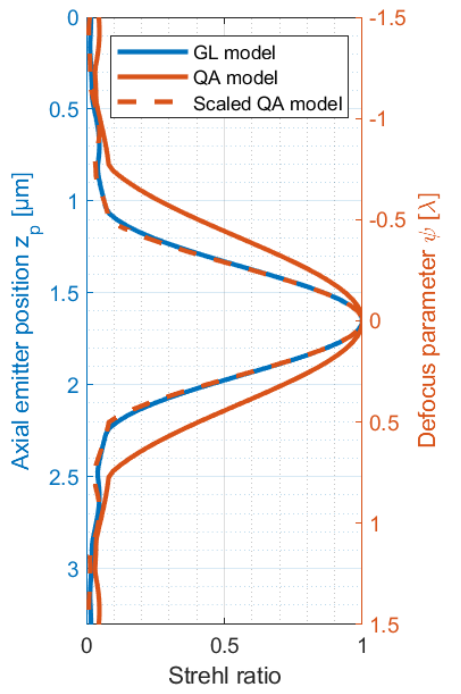

(d)

Fig. 4. (a) PSF profile as a function of the defocus parameter $\psi \in[-1.5 \lambda, 1.5 \lambda]$ using the QA model (same graph as Fig. 2.a). (b) PSF profile as a function of the emitter position $z_{\mathrm{p}}$ with the GL model $\left(n_{\mathrm{i}}=n_{\mathrm{s}}=1.33\right)$. (c) PSF profile as a function of the equivalent defocus parameter $\psi^{*}$ using the QA model. (d) Variation of the Strehl ratio for the optical systems illustrated in (a), (b) and (c). The microscope configuration and the simulation parameters are described in Table 1.

seen that the variations of the PSF and the Strehl ratio multiplied by $\beta$ are quasi-identical to those of the GL model, albeit for the slight dissymmetry on either side of the focus point.

If we substitute $z_{\mathrm{p}}$ for $z_{\max }$ (or $z_{\min }$ ) in Eq. (15), a targeted DoF range $\left[z_{\min }, z_{\max }\right]$ in the GL model is thus equivalent to a range $\left[-\psi_{\max }^{*}, \psi_{\max }^{*}\right]$ in the QA model, with

$$
\psi_{\max }^{*}=\kappa_{\psi} \frac{z_{\max }-z_{\min }}{2} .
$$

Note that this expression does not depend on the index $n_{\mathrm{i}}$ of the immersion medium. This means that the apparent scaling between the QA and GL models, observed by comparing Fig. 5.a and Fig. 5.b for example, comes from the high value of the NA in the medium of refractive index $n_{\mathrm{S}}$ and not from the refractive index mismatch between the immersion and sample media. This mismatch, on the other hand, explains the drop of the Strehl ratio and the dissymmetry of the PSF shape with respect to the focus plane.

As a conclusion, the main benefit of switching from the QA model to the GL model is to predict the actual axial shrinking of the PSF, and therefore of the DoF, in real configurations with high NA and possible index mismatch between the sample and the immersion media. In other words, it avoid overestimating the DoF range by a too optimistic model.

\section{Equivalence of GL and QA models in the presence of phase mask}

Let us now assume that a DoF-enhancing phase mask is present in the aperture stop of the microscope. As seen in Eq. (11), this amounts to adding a phase function which depends on the pupil coordinates $r$ but which is independent from the emitter position $z_{\mathrm{p}}$. Hence, if a configuration corresponding to $z_{\mathrm{p}}$ in the GL model is equivalent to a configuration corresponding to $\psi^{*}$ in the QA model - that is, they yield "equivalent" aberration wavefronts in the pupil - these aberration wavefronts should remain equivalent when using a phase mask. In consequence, we may expect that the conclusions obtained so far without mask can be extrapolated in the presence of a phase mask.
In order to verify this conjecture, we have plotted in Fig. 6 the same graphs as presented in Fig. 4 when the localization microscope is equipped with the mask shown in Fig. 3, and for $n_{\mathrm{i}}=1.52$ and $n_{\mathrm{S}}=1.33$. In order to better appreciate the variation of the PSF profile with defocus, we have changed the color bar for better contrast. We draw the same conclusions as established previously: the main difference between Fig. 6.a (QA model) and Fig. 6.b (GL model) is a global "shrinking" of the PSF variation. We have represented in Fig. 6.c the variation of the PSF of the QA model as a function of the equivalent parameter $\psi^{*}$ defined in Eq. (15), and displayed in Fig. 6.d the variations of the Strehl ratio for the configurations illustrated Fig. 6.a, Fig. 6.b and Fig. 6.c. We thus verify that even when using a phase mask, the variations of the PSF and of the Strehl ratio as a function of the equivalent parameter $\psi^{*}$ are still very similar to those of the GL model.

In the following section, we study the consequences of this approximate equivalence relation on the optimization of the DoF enhancing phase masks. Namely, we compare the masks optimized for equivalent QA and GL configurations to determine if their performance are also equivalent.

\section{EQUIVALENCE OF DOF ENHANCING MASKS OPTI- MIZED IN THE FRAMEWORK OF QA AND GL MOD- ELS}

The CRB-based phase mask optimization problem is defined in Eq. (12) and Eq. (13). It consists in minimizing the criterion $J(\rho)$, which is the maximum value of the CRB over the targeted defocus range. Since the expressions of the PSF are different in the QA and GL models, the expressions of the RCRB are also different. We thus need to define the two following criteria:

$$
\begin{gathered}
J_{\mathrm{QA}}(\boldsymbol{\rho})=\max _{\psi \in\left[-\psi_{\max }, \psi_{\max }\right]} \operatorname{RCRB}_{\mathrm{QA}}(\boldsymbol{\rho}, \psi), \\
J_{\mathrm{GL}}(\boldsymbol{\rho})=\max _{z_{\mathrm{p}} \in\left[z_{\min }, z_{\max }\right]} \operatorname{RCRB}_{\mathrm{GL}}\left(\boldsymbol{\rho}, z_{\mathrm{p}}\right),
\end{gathered}
$$




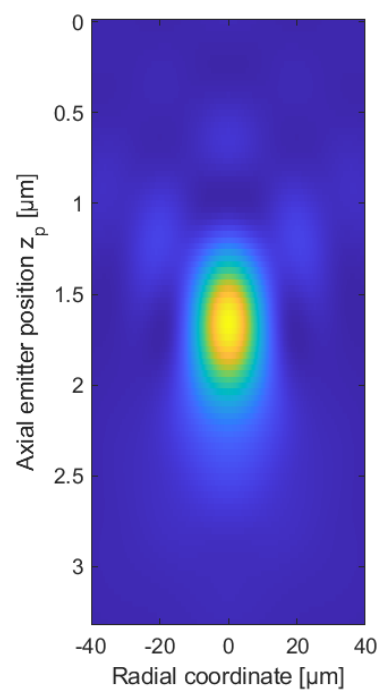

(a)

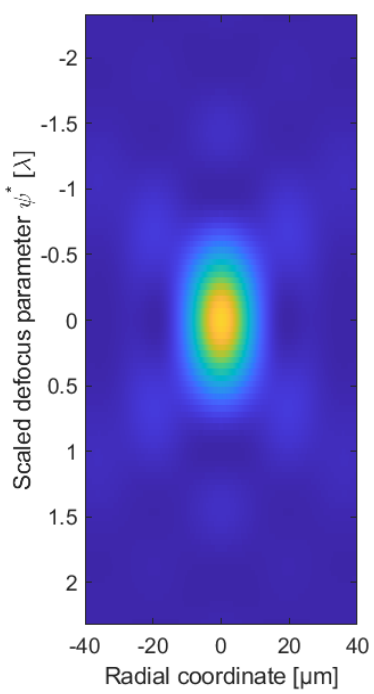

(b)

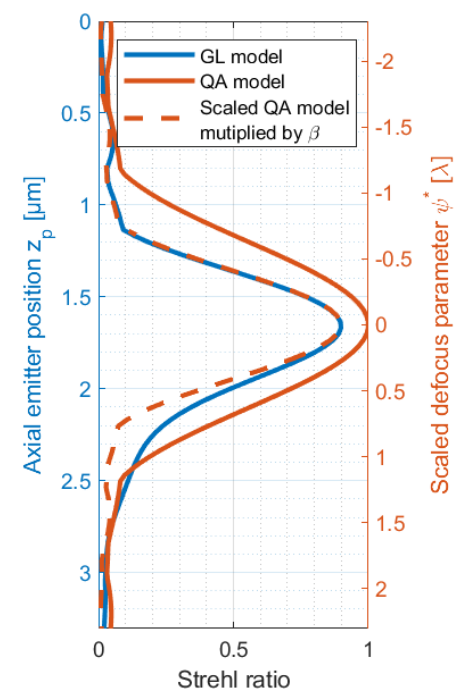

(c)

Fig. 5. (a) PSF profile as a function of the emitter position $z_{\mathrm{p}}$ with the GL model when $n_{\mathrm{i}}=1.52$ and $n_{\mathrm{s}}=1.33$. (b) PSF profile, multiplied by the correcting factor $\beta$ defined in Eq. (34), as a function of the equivalent defocus parameter $\psi^{*}$ using the QA model. (c) Variation of the Strehl ratio for the optical systems illustrated in (a) and (b). The microscope configuration and the simulation parameters are described in Table 1.

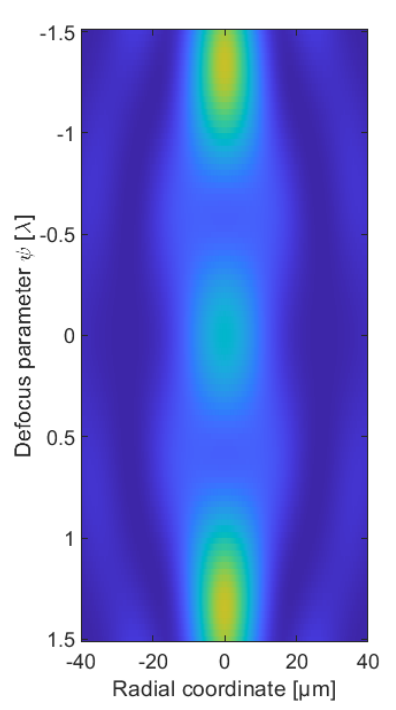

(a)

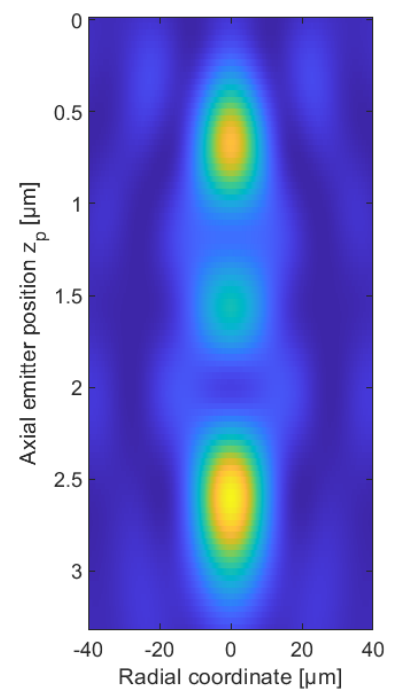

(b)

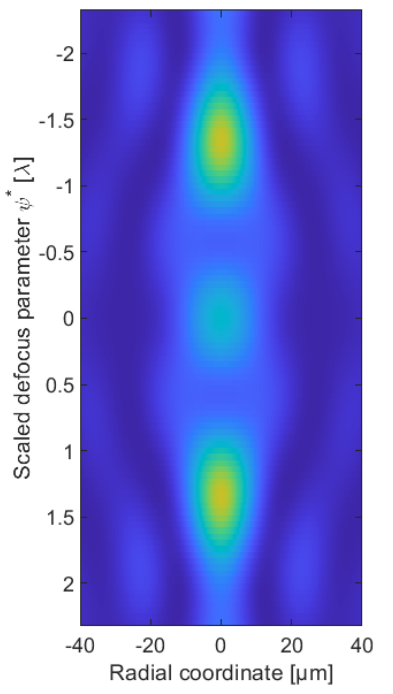

(c)

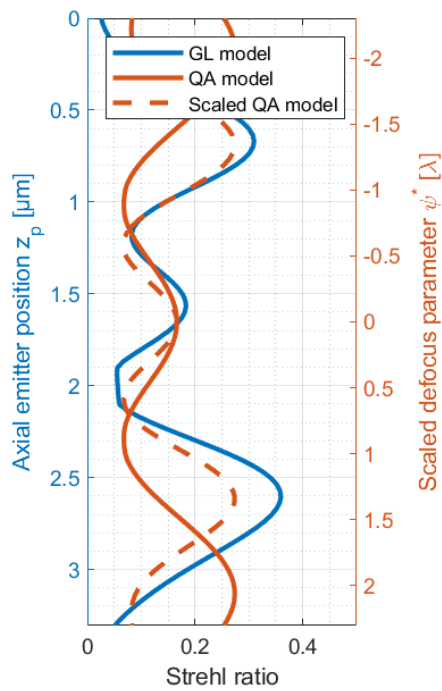

(d)

Fig. 6. (a) PSF profile as a function of the defocus parameter $\psi \in[-1.5 \lambda, 1.5 \lambda]$ using the QA model. (b) PSF profile as a function of the emitter position $z_{\mathrm{p}}$ with the GL model ( $n_{\mathrm{i}}=1.52$ and $\left.n_{\mathrm{s}}=1.33\right)$. (c) PSF profile as a function of the equivalent defocus parameter $\psi^{*}$ using the QA model. (d) Variation of the Strehl ratio for the optical systems illustrated in (a), (b) and (c). The microscope is equipped with the phase mask illustrated in Fig. 3 and its configuration and the simulation parameters are described in Table 1. 


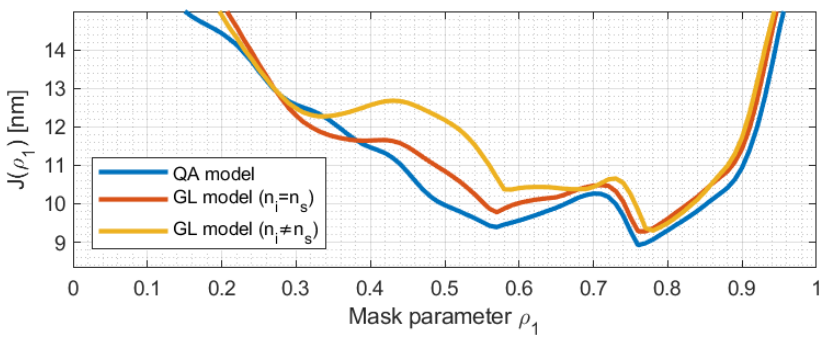

Fig. 7. Optimization landscape of 2-ring binary phase masks computed for $\psi_{\max }=1 \lambda$. We use the microscope configuration and the simulation parameters described in Table 1.

where $R C R B_{Q A}$ and $R C R B_{G L}$ are the values of the RCRB computed from the PSF expressions given by the QA model and the GL model respectively. In this section, we compare the masks obtained by optimizing these two criteria.

For this comparison, we select equivalent configurations, that is, we choose a value of $\psi_{\max }$ in the QA model, and consider the equivalent targeted DoF range in the GL model, assuming for simplicity that $z_{\min }=0$, and choosing the value of $z_{\max }$ such that $\psi_{\max }^{*}=\psi_{\max }$, where $\psi_{\max }^{*}$ is defined in Eq. (38). We will consider two possible combinations of refractive indices. In the first one, the indices of the immersion and sample media are equal: $n_{\mathrm{i}}=n_{\mathrm{s}}=1.33$. In the second one, they are different: $n_{\mathrm{i}}=1.52$ and $n_{\mathrm{s}}=1.33$. The other parameters of the imaging system are given in Table 1 .

Let us first consider a targeted DoF range $\psi_{\max }=1 \lambda$ and annular binary masks with two rings (i.e., $N=2$ ), that are defined by a single parameter denoted by $\rho_{1}$. We have represented in Fig. 7 the values of $J_{\mathrm{QA}}\left(\rho_{1}\right)$ (blue solid curve), and of $J_{\mathrm{GL}}\left(\rho_{1}\right)$ when $n_{\mathrm{i}}=n_{\mathrm{S}}$ (red solid curve) and when $n_{\mathrm{i}} \neq n_{\mathrm{s}}$ (yellow solid curve), as a function of $\rho_{1}$. The optimal values of $\rho_{1}$ correspond to the positions of the global minimum of each curve. It is observed that the curves are close to each other and that their global minima occur at very similar positions. Hence, it can be concluded that the 2-ring annular binary phase masks optimized in the framework of the QA and GL models are almost identical.

Let us now generalize this result to larger DoF ranges. For that purpose, we need to consider annular binary phase masks with larger number of rings. Indeed, as shown in Ref. [5], large DoF ranges require a larger number of rings to reach optimal localization accuracy. Therefore, in our optimization process, we consider that $N$ can be as large as 5 . Note, however, that in some cases, the performance levels-off for a smaller value of $N$, in which case the optimal mask has less than five rings. We consider three different values of the targeted DoF range: $\psi_{\max }=1 \lambda, 1.5 \lambda$, and $2 \lambda$. To optimize the criteria, which are highly non-convex, we use the particle swarm global optimization algorithm described in Ref. [21].

Table 2 synthesizes the results obtained for each considered value of $\psi_{\max }$ (first column) and combination of optical indices ( $n_{\mathrm{i}}=n_{\mathrm{s}}$ or $n_{\mathrm{i}} \neq n_{\mathrm{s}}$, second column). The third column represents the optimal masks for the GL model, defined by the parameter vector $\rho_{\mathrm{opt}}^{\mathrm{GL}}$, and the corresponding value of the criterion $J_{\mathrm{GL}}\left(\rho_{\mathrm{Opt}}^{\mathrm{GL}}\right)$, which is the maximum value of the $\mathrm{RCRB} \mathrm{GL}_{\mathrm{GL}}$ within the DoF range. It is observed that as the targeted DoF range $\psi_{\mathrm{max}}$ increases, the value of $J_{\mathrm{GL}}\left(\rho_{\mathrm{opt}}^{\mathrm{GL}}\right)$ increases, since the DoF extension problem becomes more difficult. It is also noticed that it is slightly larger in the case of $n_{\mathrm{i}} \neq n_{\mathrm{s}}$, due to the PSF alteration induced by the refractive index mismatch. The fourth column of Table 2 represents the optimal mask for the QA model, defined by the parameter vector $\rho_{\mathrm{opt}}^{\mathrm{QA}}$, and the corresponding value of the criterion $J_{\mathrm{QA}}\left(\rho_{\mathrm{opt}}^{\mathrm{QA}}\right)$. By comparison with the masks in the third column, it is seen that the masks that are optimal for QA and GL models are different but bear some similarities. In particular, the radii of their main rings are similar. The question is: are these masks equivalent in terms of DoF extension performance?

To answer this question, let us consider the following scenario: a mask has been optimized with the simple QA model, for a targeted DoF range $\psi_{\max }$, and is used in a system with high NA and possibly index mismatch, which parameters (NA, refractive indices, distances) are such that $\psi_{\max }^{*}=\psi_{\max }$. The value of the localization criterion obtained in this case, $J_{\mathrm{GL}}\left(\rho_{\mathrm{opt}}^{\mathrm{QA}}\right)$, is given in the fifth column of Table 2. By comparing the values in the third and fifth columns of the table, it is seen that $J_{\mathrm{GL}}\left(\rho_{\mathrm{opt}}^{\mathrm{QA}}\right)>J_{\mathrm{GL}}\left(\rho_{\mathrm{opt}}^{\mathrm{GL}}\right)$, which is expected since the mask $\rho_{\mathrm{opt}}^{\mathrm{QA}}$ is not optimal for the GL model. However, the values of $J_{\mathrm{GL}}\left(\rho_{\mathrm{opt}}^{\mathrm{QA}}\right)$ and $J_{\mathrm{GL}}\left(\rho_{\mathrm{opt}}^{\mathrm{GL}}\right)$ are quite close, which means that even if the mask has been optimized with the QA model, it is still close to optimality for the GL model. It is also observed in Table 2 that the departure from optimality increases with the value of $\psi_{\max }$ and with the index mismatch. However, even in the worst case, which corresponds to $\psi_{\max }=2 \lambda$ and $n_{\mathrm{i}} \neq n_{\mathrm{s}}$, one has $J_{\mathrm{GL}}\left(\rho_{\mathrm{opt}}^{\mathrm{GL}}\right)=10.25 \mathrm{~nm}$ and $J_{\mathrm{GL}}\left(\rho_{\mathrm{opt}}^{\mathrm{QA}}\right)=11.45 \mathrm{~nm}$, which corresponds to a relative difference of only $12 \%$.

As a summary, the DoF enhancing masks optimized in the framework of the QA model are also close to optimality in the framework of the GL model, provided optimization in the QA case has been done with the "equivalent" defocus parameter $\psi_{\max }^{*}$ defined in Eq. (17). This result is important since it allows the use of generic masks targeting a given $\mathrm{DoF}$ range $\psi_{\max }^{*}$ without needing precise knowledge of NA, $n_{\mathrm{i}}$ and $n_{\mathrm{s}}$. Indeed, let us consider various microscope configurations having different values of refractive indices, NA, and imaging depth, but corresponding to the same value of the reduced defocus parameter $\psi_{\max }^{*}$. The optimal masks in all these configurations yield nearly equal performance, and they are similar to the mask optimal in the framework of the QA model for a DoF range equal to $\left[-\psi_{\max }^{*}, \psi_{\max }^{*}\right]$.

\section{CONCLUSION}

In this article we have compared the DoF extending performance of phase masks optimized in the framework of the simple QA model and of the GL model that more accurately represents imaging with high NA and refractive index mismatch. We have first demonstrated that the validity of the QA model can be extended by using a scaled DoF parameter derived from the GL model. This parameter is based on the equivalence of the RMS wavefront errors. Then, we have shown that for these equivalent configurations, the optimal masks in the GL model and in the QA model are similar and yield similar localization performance. This result is important since it allows the use of generic masks without needing precise knowledge of NA, $n_{\mathrm{i}}$ and $n_{\mathrm{s}}$.

This work has many interesting perspectives. Since the annular binary phase masks are easy to manufacture, the main one would be to validate the conclusions of this paper experimentally. Moreover, the equivalent DoF parameter defined in this paper is totally independent of the type of phase mask used in the system. It would thus be interesting to apply the proposed 


\begin{tabular}{|c|c|c|c|c|c|c|}
\hline DoF & $\begin{array}{c}\text { Refractive } \\
\text { indices }\end{array}$ & & & & & \\
\hline$\psi_{\max }$ & $\left(n_{\mathrm{i}}, n_{\mathrm{S}}\right)$ & $\rho_{\mathrm{opt}}^{\mathrm{GL}}$ & $J_{\mathrm{GL}}\left(\rho_{\mathrm{opt}}^{\mathrm{GL}}\right)$ & $\rho_{\mathrm{opt}}^{\mathrm{QA}}$ & $J_{\mathrm{QA}}\left(\rho_{\mathrm{opt}}^{\mathrm{QA}}\right)$ & $J_{\mathrm{GL}}\left(\rho_{\mathrm{opt}}^{\mathrm{QA}}\right)$ \\
\hline & $n_{\mathrm{i}}=n_{\mathrm{s}}$ & & $8.32 \mathrm{~nm}$ & & & $8.55 \mathrm{~nm}$ \\
\hline & $n_{\mathrm{i}} \neq n_{\mathrm{s}}$ & & $8.42 \mathrm{~nm}$ & & & $8.90 \mathrm{~nm}$ \\
\hline & $n_{\mathrm{i}}=n_{\mathrm{s}}$ & & $9.13 \mathrm{~nm}$ & & & $9.58 \mathrm{~nm}$ \\
\hline & $n_{\mathrm{i}} \neq n_{\mathrm{s}}$ & & $9.32 \mathrm{~nm}$ & & & $10.33 \mathrm{~nm}$ \\
\hline \multirow{2}{*}{$2 \lambda$} & $n_{\mathrm{i}}=n_{\mathrm{s}}$ & & $9.87 \mathrm{~nm}$ & & \multirow{2}{*}{$10.08 \mathrm{~nm}$} & $10.82 \mathrm{~nm}$ \\
\hline & $n_{\mathrm{i}} \neq n_{\mathrm{s}}$ & & $10.25 \mathrm{~nm}$ & & & $11.45 \mathrm{~nm}$ \\
\hline
\end{tabular}

Table 2. Optimal multi-ring binary phase masks and their performance based on the criteria defined in Eq. (18). The microscope configurations and the simulation parameters are described in Table 1. 
approach to other types of phase masks, such as those aimed at 3 D localization $[3,22]$.

\section{APPENDICES}

\section{A. RMS WAVEFRONT ERROR IN THE GL MODEL}

By definition, the RMS wavefront error, denoted by $W(r)$ with $r$ the normalized radius in the pupil, is defined as

$$
\begin{aligned}
\sigma_{\mathrm{W}}^{2} & =\frac{1}{\int_{0}^{1} r \mathrm{~d} r} \int_{0}^{1}[W(r)-\bar{W}]^{2} r \mathrm{~d} r \\
& =2 \int_{0}^{1}[W(r)-\bar{W}]^{2} r \mathrm{~d} r
\end{aligned}
$$

where the average value $\bar{W}$ is defined as

$$
\bar{W}=\frac{1}{\int_{0}^{1} r \mathrm{~d} r} \int_{0}^{1} W(r) r \mathrm{~d} r=2 \int_{0}^{1} W(r) r \mathrm{~d} r .
$$

For the GL model, by substituting the OPD expression given by Eq. (14) in Eq. (19), the RMS wavefront error has the following expression:

$$
\sigma_{\mathrm{GL}}^{2}\left(z_{\mathrm{p}}, d\right)=z_{\mathrm{p}}^{2} \kappa_{\mathrm{s}}^{2}+d^{2} \kappa_{\mathrm{i}}^{2}-2 z_{\mathrm{p}} d c_{\mathrm{si}}^{2}
$$

where the coefficients $\kappa_{\mathrm{s}}^{2}, \kappa_{\mathrm{i}}^{2}$ and $c_{\mathrm{si}}^{2}$ are defined as

$$
\left\{\begin{array}{l}
\kappa_{\mathrm{s}}^{2}=2 \int_{0}^{1}\left[h_{\mathrm{s}}(r)-\overline{h_{\mathrm{s}}}\right]^{2} r \mathrm{~d} r \\
\kappa_{\mathrm{i}}^{2}=2 \int_{0}^{1}\left[h_{\mathrm{i}}(r)-\overline{h_{\mathrm{i}}}\right]^{2} r \mathrm{~d} r \\
c_{\mathrm{si}}^{2}=2 \int_{0}^{1}\left[h_{\mathrm{s}}(r)-\overline{h_{\mathrm{s}}}\right]\left[h_{\mathrm{i}}(r)-\overline{h_{\mathrm{i}}}\right] r \mathrm{~d} r
\end{array}\right.
$$

with

$$
\overline{h_{k}}=2 \int_{0}^{1} h_{k}(r) r \mathrm{~d} r \text { and } h_{k}(r)=\sqrt{n_{k}^{2}-\mathrm{NA}^{2} r^{2}},
$$

and $k$ represents the subscript letter " $\mathrm{s}$ " or " $\mathrm{i}$ " used in Eq. (22).

\section{B. OPTIMAL PARAMETER $d$ IN THE GL MODEL}

Let us assume that the targeted DoF range is $\left[z_{\min }, z_{\max }\right]$. We want to adjust the parameter $d$ so as to minimize the maximum of the RMS wavefront error $\sigma_{\mathrm{GL}}$, defined in Eq. (21), over the targeted DoF range. This leads solving the following problem:

$$
d_{\mathrm{opt}}=\arg \min _{d}\left\{\max _{z_{\mathrm{p}} \in\left[z_{\min }, z_{\max }\right]} \sigma_{\mathrm{GL}}^{2}\left(z_{\mathrm{p}}, d\right)\right\} .
$$

Since $\sigma_{\mathrm{GL}}^{2}\left(z_{\mathrm{p}}, d\right)$ is a second degree polynomial in the variable $z_{\mathrm{p}}$ with positive coefficient for the second order term (see Eq. (21)), its maximum value, for a given $d$, is reached at the extremities of the range, that is, for $z_{\mathrm{p}}=z_{\min }$ or $z_{\max }$. Thus, the problem described in Eq. (24) can be rewritten as:

$$
\sigma_{\mathrm{GL}}^{2}\left(z_{\min }, d_{\mathrm{opt}}\right)=\sigma_{\mathrm{GL}}^{2}\left(z_{\mathrm{max}}, d_{\mathrm{opt}}\right) \text {. }
$$

Solving this problem, the optimal value of $d$ is obtained as:

$$
d_{\mathrm{opt}}=\frac{z_{\min }+z_{\mathrm{max}}}{2} \frac{\kappa_{\mathrm{s}}^{2}}{c_{\mathrm{si}}^{2}} .
$$

By substituting Eq. (26) into Eq. (21) and simplifying, we obtain:

$$
\sigma_{\mathrm{GL}}^{2}\left(z_{\mathrm{p}}, d_{\mathrm{opt}}\right)=\kappa_{\mathrm{s}}^{2}\left[\left(z_{\mathrm{p}}-z_{\text {focus }}\right)^{2}+\alpha z_{\text {focus }}^{2}\right]
$$

with

$$
z_{\text {focus }}=\frac{z_{\min }+z_{\max }}{2} \quad \text { and } \quad \alpha=\frac{\kappa_{\mathrm{s}}^{2} \kappa_{\mathrm{i}}^{2}}{c_{\mathrm{si}}^{4}}-1 .
$$

\section{SCALING BETWEEN THE QA AND GL MODELS}

It is interesting to compare Eq. (27) to the expression of the RMS wavefront error in the QA model, obtained by substituting the OPD expression given by Eq. (4) and Eq. (5) into Eq. (19):

$$
\sigma_{\mathrm{QA}}^{2}=\frac{\psi^{2}}{12}=\kappa_{\mathrm{QA}}^{2}\left(z_{\mathrm{p}}-z_{\mathrm{focus}}\right)^{2}
$$

with

$$
\kappa_{\mathrm{QA}}^{2}=\frac{\mathrm{NA}^{4}}{48 n_{\mathrm{S}}^{2}} .
$$

One observes that Eq. (27) and Eq. (29) are both quadratic functions of the variable $\Delta z=\left(z_{\mathrm{p}}-z_{\text {focus }}\right)$, but they bear two important differences. The first one is that the coefficients of the quadratic term are different in the two equations. For the QA model, it is $\kappa_{\mathrm{QA}}$, and for the GL model, it is $\kappa_{\mathrm{S}}$ whose expression is given in Eq. (22). With the help of a symbolic math software, we were able to determine the first terms of the Taylor series expansion of $\kappa_{\mathrm{S}}^{2}$ with respect to $\left(\mathrm{NA} / n_{\mathrm{S}}\right)^{2}$ :

$$
\kappa_{\mathrm{s}}^{2} \simeq \frac{\mathrm{NA}^{4}}{48 n_{\mathrm{s}}^{2}}\left(1+\frac{\mathrm{NA}^{2}}{2 n_{\mathrm{s}}^{2}}+\frac{7 \mathrm{NA}^{4}}{24 n_{\mathrm{s}}^{4}}+\frac{3 \mathrm{NA}^{6}}{16 n_{\mathrm{s}}^{6}}\right) .
$$

Note that the first term is exactly $\kappa_{\mathrm{QA}}^{2}$, which shows that for a low NA, both models predict, as expected, the same behavior. However, in the general case, the QA and GL models lead to the same value of the RMS wavefront error for different depth variations $\Delta z$, given by $\kappa_{\mathrm{QA}}^{2} \Delta z_{\mathrm{QA}}^{2}=\kappa_{\mathrm{S}}^{2} \Delta z_{\mathrm{GL}}^{2}$. We can therefore introduce a scaling factor:

$$
\begin{aligned}
\eta & =\frac{\Delta z_{\mathrm{QA}}}{\Delta z_{\mathrm{GL}}}=\frac{\kappa_{\mathrm{s}}}{\kappa_{\mathrm{QA}}} \\
& \simeq 1+\frac{1}{4}\left(\frac{\mathrm{NA}}{n_{\mathrm{s}}}\right)^{2}+\frac{11}{96}\left(\frac{\mathrm{NA}}{n_{\mathrm{s}}}\right)^{4}+\frac{25}{384}\left(\frac{\mathrm{NA}}{n_{\mathrm{s}}}\right)^{6}
\end{aligned}
$$

It is easily seen that this factor starts at 1 for low (zero) NA and significantly grows as NA increases. This means that when NA is large, the QA model yields a too optimistic value of the DoF extension. According to the GL model, this value has to be divided by the the factor $\eta$ as NA increases. This is clearly seen by comparing Fig. 4.a and Fig. 4.b for example. Interestingly, this scaling coefficient depends only on $n_{\mathrm{S}}$ (through $\kappa_{\mathrm{S}}$ ), and not on $n_{\mathrm{i}}$. It is thus independent of the index mismatch between the immersion and sample media: it is due only to the high value of $\mathrm{NA}$ in the medium with refractive index $n_{\mathrm{s}}$.

The second difference difference between Eq. (27) and Eq. (29) is the presence of an offset term equal to $\alpha \kappa_{\mathrm{s}}^{2} z_{\text {focus }}^{2}$ in $\sigma_{\mathrm{GL}}^{2}$. According to the Cauchy-Schwartz inequality, $\kappa_{\mathrm{s}} \kappa_{\mathrm{i}} \geq c_{\mathrm{si}}^{2}$, the factor $\alpha$ is thus positive or zero. Moreover, this term vanishes when $n_{\mathrm{i}}=n_{\mathrm{s}}$, since in this case $\alpha=0$, and increases with index mismatch and the value of $z_{\text {focus }}$.

To illustrate the effects of these two differences, let us consider the Strehl ratio, denoted by $S$, which is the ratio between the maximal values of the actual PSF and of the Airy pattern for the same aperture. A rule of thumb widely used in optical system design states that if the RMS wavefront error $\sigma$ is not too large, the Strehl ratio can be expressed as [23]:

$$
S \simeq \exp \left[-\left(\frac{2 \pi}{\lambda} \sigma\right)^{2}\right]
$$

Hence, in case of index mismatch, if one compares the variation of $S$ with $z_{\mathrm{p}}$ in the GL model to that in the QA model, one 
should observe an axial shrinking by the coefficient $\eta$ and a global multiplication by a factor

$$
\beta=\exp \left[-\left(\frac{2 \pi}{\lambda}\right)^{2} \alpha \kappa_{\mathrm{s}}^{2} z_{\text {focus }}^{2}\right] .
$$

Fig. 5.c. shows an excellent agreement with this predicted behavior.

\section{EQUIVALENT VALUE OF $\psi$}

It can be noted that even if $n_{\mathrm{s}} \neq n_{\mathrm{i}}$, the offset term in Eq. (27) is very small for the parameters used in our simulations of localization microscopy: the standard values of $n_{\mathrm{s}}$ and of $n_{\mathrm{i}}$ in Table 1 lead to $\alpha=0.0062$, and since emitters lie only a few micrometers below the coverslip, $z_{\text {focus }}$ is small. If this offset term is neglected, Eq. (27) becomes:

$$
\sigma_{\mathrm{GL}}^{2}\left(z_{\mathrm{p}}, d_{\mathrm{opt}}\right) \simeq \kappa_{\mathrm{s}}^{2}\left(z_{\mathrm{p}}-z_{\text {focus }}\right)^{2}
$$

By equalizing this expression with the RMS wavefront error of the QA model defined in Eq. (29), we can define the value $\psi^{*}$ of the defocus parameter in the QA model that is "equivalent" to an emitter position $z_{\mathrm{p}}$ in the GL model:

$$
\psi^{*}=\kappa_{\psi}\left(z_{\mathrm{p}}-z_{\text {focus }}\right),
$$

with

$$
\kappa_{\psi}=2 \sqrt{3} \kappa_{\mathrm{s}} \simeq \frac{\mathrm{NA}^{2}}{2 n_{\mathrm{s}}}+\frac{\mathrm{NA}^{4}}{8 n_{\mathrm{s}}^{3}}+\frac{11 \mathrm{NA}^{6}}{192 n_{\mathrm{s}}^{5}}+\frac{25 \mathrm{NA}^{8}}{768 n_{\mathrm{s}}^{7}} .
$$

Replacing $z_{\mathrm{p}}$ with $z_{\max }$ or $z_{\min }$ in Eq. (36), one can also define the equivalent targeted DoF range as

$$
\psi_{\max }^{*}=\kappa_{\psi} \frac{z_{\max }-z_{\min }}{2} .
$$

Eq. (38) makes it possible to establish a simple correspondence between the targeted DoF ranges in the GL and QA models.

Acknowledgments. This work has received the support of the French research group GdR ISIS of the CNRS through the Projet de Recherche Exploratoire MASK.

Disclosures. The authors declare no conflicts of interest.

\section{REFERENCES}

1. S. Ram, P. Prabhat, J. Chao, E. Sally Ward, and R. J. Ober, "High Accuracy 3D Quantum Dot Tracking with Multifocal Plane Microscopy for the Study of Fast Intracellular Dynamics in Live Cells," Biophys. J. 95, 6025-6043 (2008).

2. S. R. P. Pavani, M. A. Thompson, J. S. Biteen, S. J. Lord, N. Liu, R. J. Twieg, R. Piestun, and W. E. Moerner, "Three-dimensional, singlemolecule fluorescence imaging beyond the diffraction limit by using a double-helix point spread function," Proc. Natl. Acad. Sci. 106, 29952999 (2009).

3. Y. Shechtman, S. J. Sahl, A. S. Backer, and W. E. Moerner, "Optimal Point Spread Function Design for 3D Imaging," Phys. Rev. Lett. 113, 133902 (2014).

4. O. Lévêque, C. Kulcsár, H. Sauer, A. Lee, P. Bon, L. Cognet, and F. Goudail, "Can phase masks extend depth-of-field in localization microscopy?" in Unconventional Optical Imaging II, vol. 11351 C. Fournier, M. P. Georges, and G. Popescu, eds., International Society for Optics and Photonics (SPIE, 2020), pp. $50-58$.

5. O. Lévêque, C. Kulcsár, A. Lee, H. Sauer, A. Aleksanyan, P. Bon, L. Cognet, and F. Goudail, "Co-designed annular binary phase masks for depth-of-field extension in single-molecule localization microscopy," Opt. Express 28, 32426-32446 (2020).
6. J. W. Goodman, Introduction to Fourier optics (W.H. Freeman \& Company, 2017), 4th ed.

7. S. F. Gibson and F. Lanni, "Experimental test of an analytical model of aberration in an oil-immersion objective lens used in three-dimensional light microscopy," J. Opt. Soc. Am. A 9, 154-166 (1992).

8. F. Aguet, D. V. D. Ville, and M. Unser, "A maximum-likelihood formalism for sub-resolution axial localization of fluorescent nanoparticles," Opt. Express 13, 10503-10522 (2005).

9. J. J. M. Braat, P. Dirksen, A. J. E. M. Janssen, and A. S. van de Nes, "Extended Nijboer-Zernike representation of the vector field in the focal region of an aberrated high-aperture optical system," J. Opt. Soc. Am. A 20, 2281-2292 (2003).

10. F. Aguet, "Super-Resolution Fluorescence Microscopy Based on Physical Models," Ph.D. thesis, École polytechnique fédérale de Lausanne (2009).

11. S. M. Kay, Fundamentals of Statistical Signal Processing: Estimation theory, Prentice Hall signal processing series (Prentice-Hall PTR, 1993).

12. R. J. Ober, S. Ram, and E. S. Ward, "Localization Accuracy in SingleMolecule Microscopy," Biophys. J. 86, 1185 - 1200 (2004).

13. E. R. Dowski and W. T. Cathey, "Extended depth of field through wavefront coding," Appl. Opt. 34, 1859-1866 (1995).

14. S. S. Sherif, W. T. Cathey, and E. R. Dowski, "Phase plate to extend the depth of field of incoherent hybrid imaging systems," Appl. Opt. 43, 2709-2721 (2004).

15. A. Sauceda and J. Ojeda-Castañeda, "High focal depth with fractionalpower wave fronts," Opt. Lett. 29, 560-562 (2004).

16. Q. Yang, L. Liu, and J. Sun, "Optimized phase pupil masks for extended depth of field," Opt. Commun. 272, 56 - 66 (2007).

17. N. Caron and Y. Sheng, "Polynomial phase masks for extending the depth of field of a microscope," Appl. Opt. 47, E39-E43 (2008).

18. F. Zhou, G. Li, H. Zhang, and D. Wang, "Rational phase mask to extend the depth of field in optical-digital hybrid imaging systems," Opt. Lett. 34, 380-382 (2009).

19. A. Chomik, "Déconvolution 3D orientée vers la reconstruction d'objets biologiques observés en microscopie optique de fluorescence," Ph.D. thesis, Universite de Haute-Alsace (1997).

20. A. J. E. M. Janssen, "Extended Nijboer-Zernike approach for the computation of optical point-spread functions," J. Opt. Soc. Am. A 19, 849-857 (2002).

21. J. Kennedy and R. Eberhart, "Particle swarm optimization," in Proceedings of ICNN'95 - International Conference on Neural Networks, vol. 4 (1995), pp. 1942-1948 vol.4.

22. S. R. P. Pavani and R. Piestun, "High-efficiency rotating point spread functions," Opt. Express 16, 3484-3489 (2008).

23. V. N. Mahajan, "Strehl ratio for primary aberrations in terms of their aberration variance," J. Opt. Soc. Am. 73, 860-861 (1983). 